\title{
Documentation of the Corrosion of Composite-Extruded Aluminium Matrix Extrusions using the Push-Out Test
}

\author{
M. Merzkirch ${ }^{1, a}$, K. A. Weidenmann ${ }^{1, b}$, E. Kerscher ${ }^{1, c}$ and D. Löhe ${ }^{1}$ \\ ${ }^{1}$ Institut für Werkstoffkunde I, Universität Karlsruhe (TH), Kaiserstr. 12, 76131 Karlsruhe, Germany

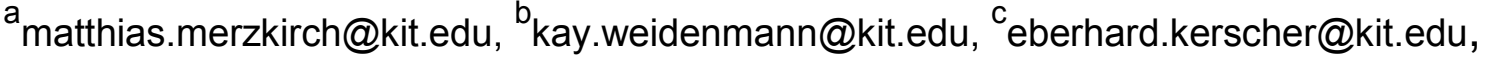 \\ cotlef.loehe@kit.edu
}

Keywords: Push-out test, corrosion, metal matrix composites, composite-extrusion

\begin{abstract}
A possibility to increase both stiffness and strength of aluminium-based structures for the application in lightweight profiles for vehicle space frames is the use of composite extrusions in which high-strength metallic reinforcements are incorporated. Within the scope of the present investigations, composite-extruded profiles with wire-reinforcements made of austenitic spring steel 1.4310 (X10CrNi18-8), in an aluminium matrix AA6060 (AlMgSi0.5), which were exposed to different corrosive media for different times, were characterised in terms of the debonding shear strength using the push-out-technique. The formation of a galvanic couple could be conceived mathematically in regard of terms describing the formation of a shear-impeding layer and the corrosive attack. Thereby the parameters for the different media could be determined.
\end{abstract}

\section{Introduction}

Aluminium and its alloys are highly qualified candidate materials for lightweight automotive constructions due to their low density in addition to the outstanding formability. If the designed space is limited, reinforced aluminium-matrix-based profiles present an attractive solution to increase both specific stiffness and specific strength. The bar extrusion technology represents a promising alternative to conventional casting techniques and is a solid state manufacturing route for composites. This can be realized in two different ways: first, the regular extrusion of discontinuously reinforced composite billets or, secondly, the composite extrusion of regular billets using modified extrusion dies which allow an in situ continuous reinforcement during the extrusion process. In the welding chamber of the extrusion press, the reinforcing elements, e.g. wires or ropes, are embedded into the matrix under high pressure at high temperature with controlled position inside the profile to optimize the load capacity [1].

Wire-reinforced composite-extruded aluminium matrix composites are appreciated as an efficient material concept. However, corrosive attack between the relatively basic matrix alloy and the electrochemically more noble reinforcing elements as a result of the electrically conductive contact could be a problem. Due to the formation of a galvanic couple, the interface between the composite components which is responsible for the internal load transfer between matrix and reinforcing component and finally important for the mechanical capability of the composite material is susceptible to enhanced corrosive attack.

In addition to the qualitative evaluation of the interface and the damage due to the corrosive load by means of classical metallographic analysis, the push-out test, first proposed by Marshall [2], offers a possibility to measure quantitatively the shear strength of the composite interface and its change as a function of time. By means of an indenter, one single reinforcement is loaded axially until damage of the interface occurs. Ideally the interface only experiences shear loads. Actually, due to the lateral expansion by the compression load, a certain normal stress occurs [3]. Neglecting this, the maximal debonding shear strength is calculated through the load maximum of the loaddisplacement curve (Fig. 1), 


$$
\sigma_{\text {deb }}=\frac{F_{\max }}{\pi \cdot d \cdot h}
$$

in the case of thin samples and by knowledge of the diameter of the reinforcing element and the thickness of the sample $[3,4]$.
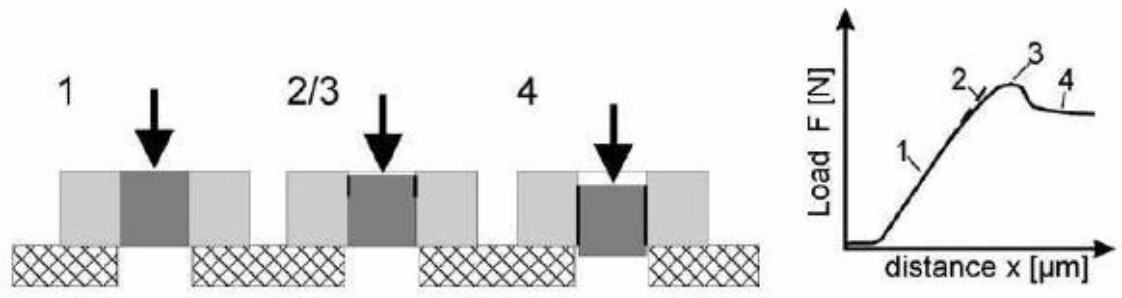

1. linear increase of load, interface stays intact

2. Stepwise debonding of interface

3. complete debonding $\rightarrow$ debonding shear strength

4. Reinforcement is pushed out $\rightarrow$ frictional shear stress

Figure 1:Functional principle and schematic load-displacement graph of a fibre push-out-test [4]

According to [5] the degree of corrosive damage can be quantified by the weight loss per time or the rate of surface attack. This is also valid for the change of tensile and fracture strength or yield strength with time. Within this work the time-dependent change of the debonding shear strength serves as a measure for damage evolution.

Based on a constant rate of corrosion with a simultaneous consideration of an eventual increase in the debonding shear strength by the formation of an oxide layer, which impedes the shearing, the following mathematical approach for the description of the change of the debonding shear strength is proposed:

$$
\sigma_{\text {deb }}(t)=\sigma_{\text {deb }}^{0}+A \cdot t^{n}-B \cdot t
$$

Here, $\sigma_{\text {deb }}^{0}$ is the debonding shear strength at the beginning (without a corrosive load). The term $\mathrm{A} \cdot \mathrm{t}^{\mathrm{n}}$ describes the potential formation of a shear-impeding layer; the term $\mathrm{B} \cdot \mathrm{t}$ describes a linear corrosion.

\section{Experimental}

Sample Materials. The analysed composite profiles with a rectangular cross section of $56 \times 5 \mathrm{~mm}^{2}$ were produced on a $10 \mathrm{MN}$ bar extrusion press modified for composite extrusion. The profile was tempered in air directly after the bar extrusion thereby age hardening the aluminium matrix AA6060 (AlMgSi0.5) (naturally aged state T4). The reinforcing wires, made from stainless austenitic spring steel, 1.4310 (X10CrNi 18-8) had a diameter of $1 \mathrm{~mm}$. The wires were cleaned with acetone prior to the bar extrusion. The ram speed of the plunger of the bar extrusion machine was $1 \mathrm{~mm} / \mathrm{s}$ at a press ratio of 1:60. The samples for the push-out test, which were cut on a precision cut-off machine, had a thickness of $1 \mathrm{~mm}$.

Experimental setup. The corrosion tests were realised in special multi compartment cases. Fig. 2 shows the experimental setup. The outer case was filled with the corrosive medium in which compressed air was blown through a frit to assure an adequate bath movement. A smaller inner case, having many holes to assure the circulation of the medium and in which the push-out samples were situated, hung in the large outer case. 


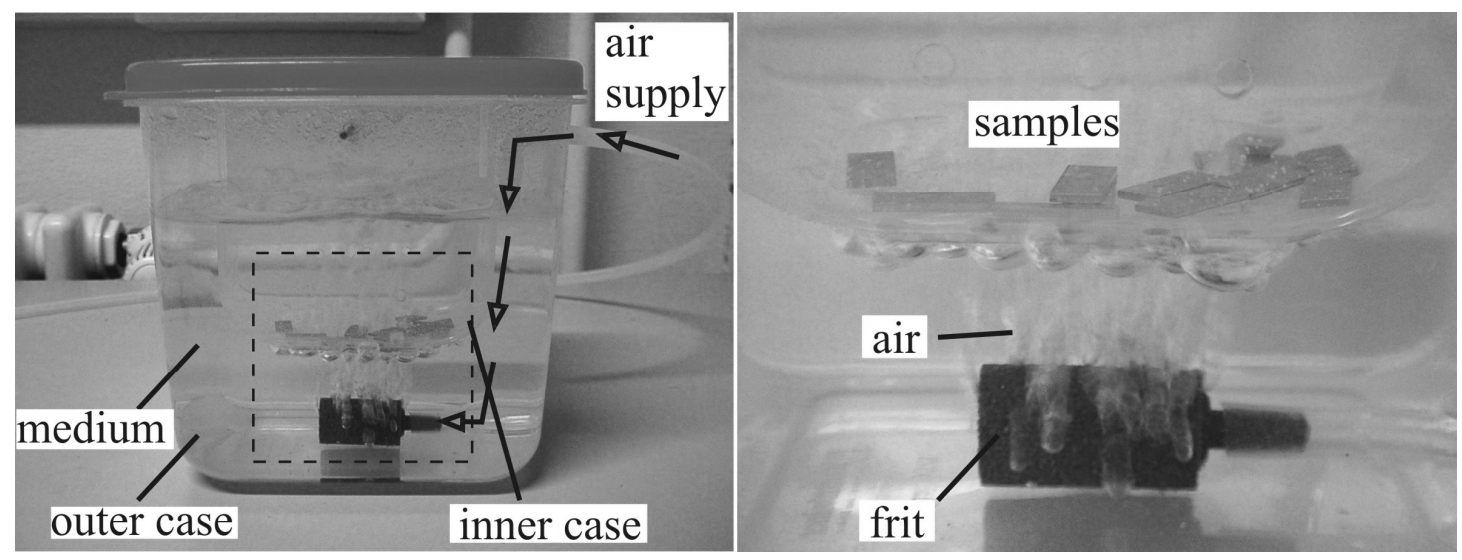

Figure 2: Multiple chamber case for corrosion tests: overview (left), detailed exposure of the periphery of the sample

Four corrosive media were used: commercial deionised water $\left(\mathrm{H}_{2} \mathrm{O}\right)$, a $2 \%$ common salt solution $(\mathrm{NaCl})$, a $2 \%$ caustic soda solution $(\mathrm{NaOH})$ and a street dirt suspension after DIN ISO 9462 (street) [6]. The last one also contained $0.6 \%$ common salt and served for the simulation of a contamination, as it appears for the application of composite materials in road traffic. The caustic soda was chosen to attack the aluminium. The common salt should mainly act on the spring steel, which is, due to its composition, not saltwater-proof. The deionised water served as a neutral reference solution. The air stream through the chamber was regulated with an analogue flow measuring device to constant $3 \mathrm{l} / \mathrm{min}$. Altogether 36 samples were exposed to each medium. 12 samples each were taken out after $10 \mathrm{~h}, 100 \mathrm{~h}$ and $1000 \mathrm{~h}$, and the debonding shear strength was characterised with the push-out-test. For determining $\sigma_{\text {deb }}^{0}$, the debonding shear strength of samples without any corrosive load was measured. The push-out tests were carried out on a universal testing machine because of the relatively large diameter of the wire requiring high loads. The universal testing machine had a registration hardness probe with a cone shaped indenter and a calotte point [7]. From the measured force maximum the debonding shear strength was determined with Eq. 1. Directly after reaching the decrease of the force (see Fig. 1) the test was interrupted to avoid failure of the interface region by penetration of the cone shaped indenter. The damaged push-out samples were investigated metallographically.

\section{Results}

Change of the debonding shear strength. The change of the debonding shear strength depending on the exposure time to the medium is shown in Fig. 3. It is noticeable that for every medium the debonding shear strength values after $10 \mathrm{~h}$ of exposure time in relation to the reference debonding shear strength $\sigma_{\text {deb }}^{0}$ increase slightly. After $100 \mathrm{~h}$ only the debonding shear strength values of in $\mathrm{NaOH}$ exposed samples decrease in relation to the value of $10 \mathrm{~h}$. After $1000 \mathrm{~h}$ the samples which were exposed to deionised water show only slight changes while the reduction of the debonding shear strength of the other samples is significant. The debonding shear strength of those samples which were exposed to $\mathrm{NaOH}$ could not be determined because the aluminium matrix was completely severed. 

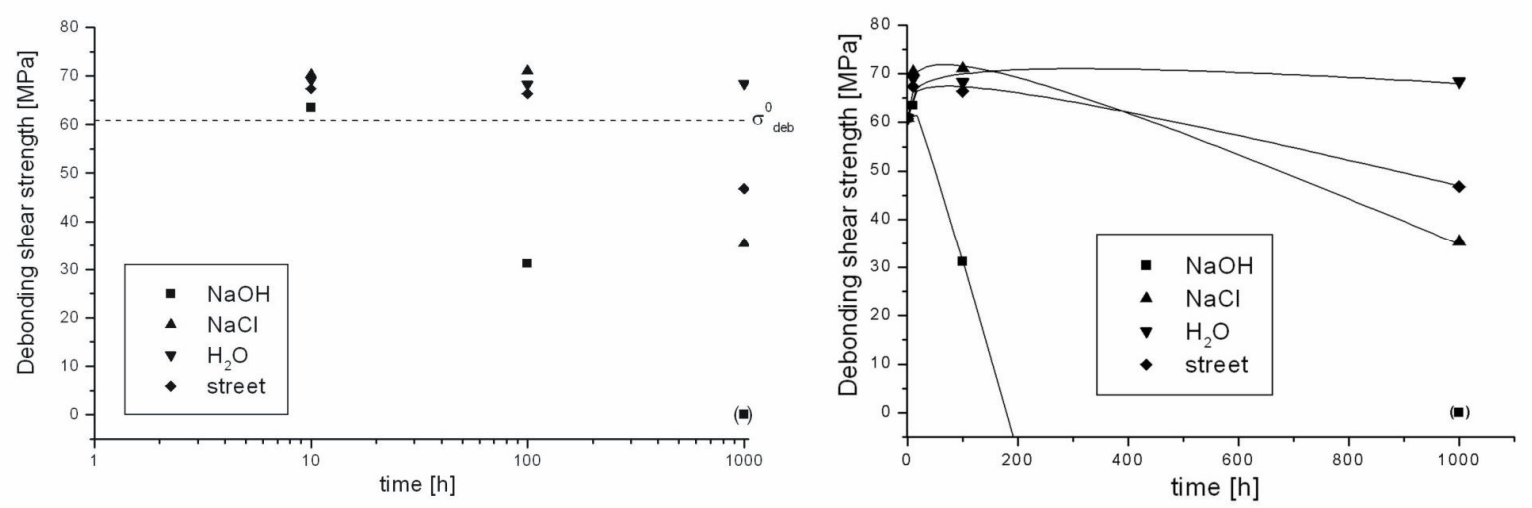

Figure 3: Averages of the debonding shear strength of the spring steel reinforced profiles after being exposed to different media (logarithmic scale, left side) and interpolated curve (linear scale, right side).

The evolution of the debonding shear strength was described mathematically with a parabolic growth of the oxide layer $(\mathrm{n}=0.5)$, at first. However, Table 1 shows that the corrosion rate $\mathrm{B}$ is influenced by the growth of the oxide layer, see Eq. 2 .

Table 1: Parameters for the description of the debonding shear strength

\begin{tabular}{l|lc|ll}
\hline Medium & \multicolumn{3}{|c|}{$\mathrm{n}=0.5$} & \multicolumn{2}{c}{$\mathrm{n}=0.25$} \\
\hline & $\mathrm{A}$ & $\mathrm{B}$ & $\mathrm{A}$ & $\mathrm{B}$ \\
$\mathrm{H}_{2} \mathrm{O}$ & 1.24 & 0.03 & 3.24 & 0.01 \\
$\mathrm{NaOH}$ & 2.26 & 0.52 & 3.83 & 0.42 \\
$\mathrm{NaCl}$ & 2.07 & 0.02 & 5.16 & 0.06 \\
street & 1.19 & 0.05 & 3.05 & 0.03 \\
\hline
\end{tabular}

Indeed the medium $\mathrm{NaOH}$ has the highest $\mathrm{B}$-value (the strongest decrease), which is approved by Fig. 3. In comparison to the B-value of the distilled water and the street dirt suspension the value of the medium $\mathrm{NaCl}$ seems to be too small. The presumed linear corrosion process is better described when a parabolic growth of order $4(\mathrm{n}=0.25)$ is assumed. There, the corrosion rate $\mathrm{B}$ increases in the range $\mathrm{H}_{2} \mathrm{O}$ - street $-\mathrm{NaCl}-\mathrm{NaOH}$, as observed for the debonding strength in the push-out tests. The linearly applied data is shown in Fig. 3 and interpolated with the help of the parameters in Table $1(n=0.25)$. For the calculation of the above mentioned parameters the value of the medium $\mathrm{NaOH}$ for $1000 \mathrm{~h}$ is disregarded, because no debonding shear strength was measured.

A good accordance between the measured data and the interpolated curves is given with Eq. 2 using $\mathrm{n}=0.25$.

Metallographic investigations. Comparing the surfaces of the samples which were exposed for 10 $\mathrm{h}$, every sample type shows the same. Compared to an unexposed sample the surfaces of all exposed samples are tarnished. Fig. 4 shows this with an example of 2 samples, which were exposed for $10 \mathrm{~h}$ to a common salt solution and a street dirt suspension, respectively. 


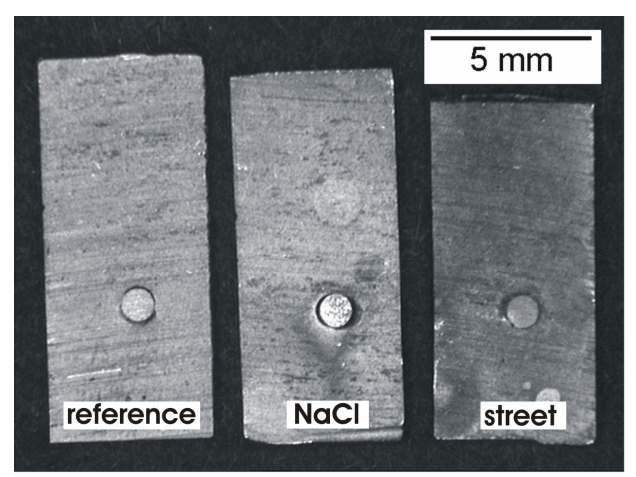

Figure 4:Macroscopic images of the surfaces of the samples after $0 \mathrm{~h}$ exposure time (left) and after $10 \mathrm{~h}$ exposure time to $\mathrm{NaCl}$ (middle) und street dirt suspension (right).

The development of the damage is shown in transverse sections prepared after the push-out tests. As expected, the damage is concentrated on the interface between wire and matrix $[8,9]$. The wire itself does not show any corrosion traces in any medium. Far from the interface the matrix is only slightly corroded, with the exception of samples exposed to $\mathrm{NaOH}$. There, a global etch attack comes up on the aluminium, which finally leads to a dissolution. Fig. 5 shows exemplarily the development of the damage on samples exposed to $\mathrm{NaCl}$.
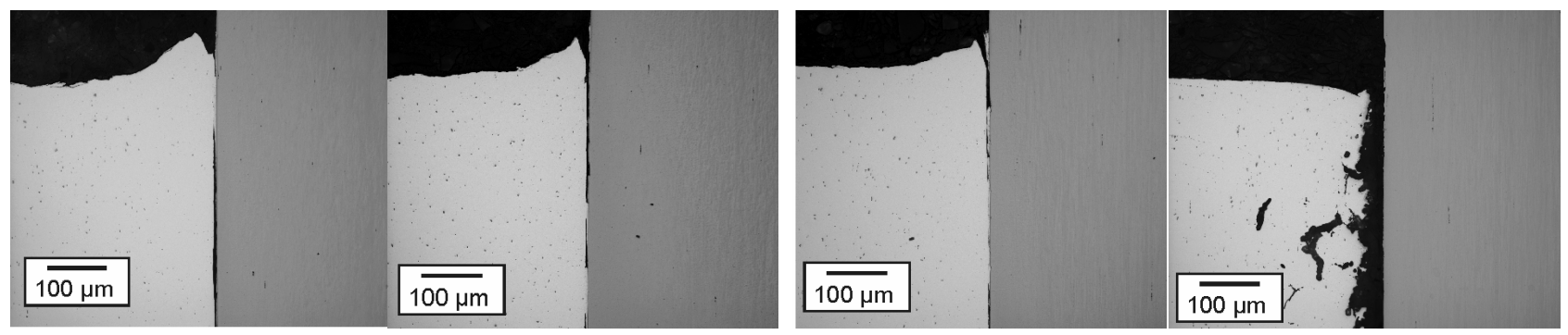

Figure 5: Transverse sections of pushed out samples after $0 \mathrm{~h}$ exposure time and after $10 \mathrm{~h}$, $100 \mathrm{~h}$ and $1000 \mathrm{~h}$ exposure time to $\mathrm{NaCl}$ (f.l.t.r.).

Additionally, the comparison of a sample exposed $100 \mathrm{~h}$ to deionised water with a sample exposed to $\mathrm{NaOH}$ for the same time shows the degree of damage, Fig. 6.
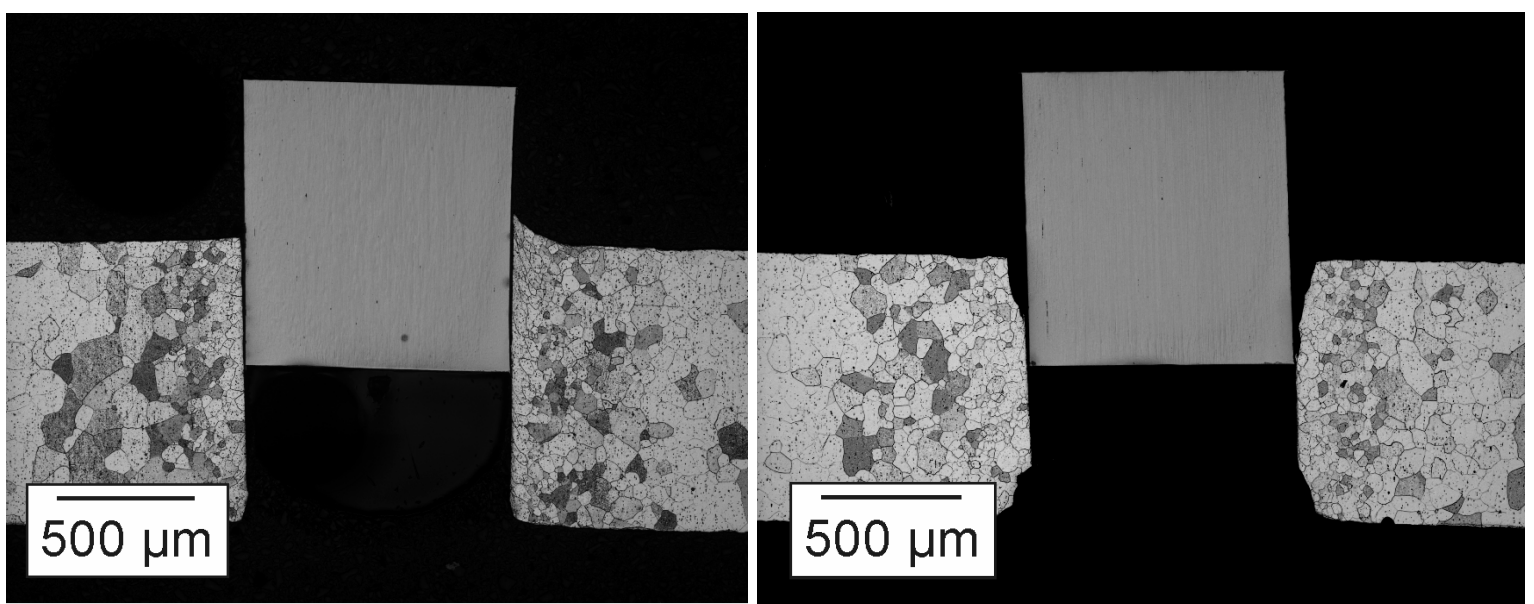

Figure 6: Transverse sections of pushed out samples after $100 \mathrm{~h}$ exposure time to $\mathrm{H}_{2} \mathrm{O}$ (left) and to $\mathrm{NaOH}$ (right). 


\section{Summary}

The investigations showed that corrosion damage appears in the investigated compounds by forming a galvanic couple between the matrix (AA6060) and the wires (1.4310) during the exposure to different media according to [8,9]. This can be quantified with the push-out test. Thereby the damage or the corrosion rate respectively is dependent on the used medium and increases in the range $\mathrm{H}_{2} \mathrm{O}-$ street $-\mathrm{NaCl}-\mathrm{NaOH}$. The change of the debonding shear strength with the exposure time could be quantitatively expressed by

$$
\sigma_{\text {deb }}(t)=\sigma_{\text {deb }}^{0}+A \cdot t^{0,25}-B \cdot t
$$

For every medium the debonding shear strength increases at first. This may be ascribed to the influence of the forming tarnishing layer, which is very thin and hardly to recognize under the light microscope. By comparison of the medium street dirt suspension and $\mathrm{NaCl}$ with respect to the corrosion rate $\mathrm{B}$, a ratio of 1:2 exists, whereupon the ratio between the $\mathrm{NaCl}$ concentrations is 1:3.33. The damage must be led back to other mechanisms in the case of the street dirt suspension. All in all, only long exposure times or a very strong corrosion impact of the medium $(\mathrm{NaOH})$ lead to a clear reduction of the debonding shear strength. The decrease can be traced back to the removal of the matrix material. In the case of the sample which was exposed $100 \mathrm{~h}$ to $\mathrm{NaOH}$, the supporting interface had only half of its initial length - consistently the debonding shear strength should be reduced to about $50 \%$, as was approved by the measurements.

Overall, it can be summarized that the damage starts from the front surface - and therefore on a real lightweight structure of composite profiles from the exposed side. Therefore the critical damage of the bearing structure through corrosion is excluded or avoidable by protective coatings.

\section{Acknowledgements}

This paper is based on investigations of the Transregional Collaborative Research Centre SFB/TR10, which is kindly supported by the German Research Foundation (DFG).

\section{References}

[1] M. Kleiner, M. Schomäcker, M. Schikorra, A. Klaus, Mat.-wiss. u. Werkst. Vol. $35 / 7$ (2004), p. 431-439.

[2] D. B. Marshall, J. Am. Ceram. Soc. Vol. 67 (1984), C259-260.

[3] T. W. Clyne, P. J. Withers, An Introduction to Metal Matrix Composites, Cambridge University Press, Cambridge, Great Britain, (1993)

[4] J. Janczak, R. Stackpole, G. Bürki, L. Rohr, 17th Int. SAMPE. Basel, Schweiz, (1996)

[5] E. Mattsson, Basic Corrosion Technology for Scientists and Engineers, IOM Communications, London, Great Britain, (2001)

[6] DIN ISO 9462, Skibindungen für den alpinen Skilauf - Sicherheitstechnische Anforderungen und Prüfungen, Beuth-Verlag, Berlin, (2004)

[7] K. A. Weidenmann, C. Fleck, V. Schulze, D. Löhe, in: M. Schlimmer, Verbundwerkstoffe und Werkstoffverbunde, DGM-Matinfo-Verlag, 45-50, (2005)

[8] J. J. Theler, A. Wagner, A. Ames, Herstellung von Aluminium/Stahl-Verbundstromschienen mit metallurgischer Bindung, Metallwissenschaft. und Technik 3, 223-227, (1976)

[9] A. Wagner, U. Hodel, Aluminium-/Stahl-Verbundprofil mit metallischer Bindung zwischen Stahl und Aluminium, Metallwisschenschaft und Technik [2] 147-151, (1979)75 